\title{
RESIDUAL STRESS DETERMINATION IN THE CEMENTITE AND FERRITE PHASES OF HIGH CARBON STEEL
}

\author{
P. VAN HOUTTE ${ }^{a, *}, K_{\text {. VAN ACKER }}{ }^{b}$ and J. ROOT ${ }^{c}$ \\ ${ }^{\mathrm{a}}$ Department MTM, Katholieke Universiteit Leuven, Belgium; ${ }^{\mathrm{b}}$ AIB-Vincotte \\ Inter, Brussels, Belgium; ' $A E C L$, Chalk River, Ontario, Canada
}

The paper describes a study of the residual stress and the texture in cold drawn wires of pearlitic high carbon steel. Total true strains in wire drawing ranged from 1.96 to 2.59 . Such material consists of a ferrite and a cementite phase, both in lamellar form. First, a discussion is given as to the various types of residual stresses which can be expected in such a material (macrostresses, grain microstresses, phase microstresses and their components). Next the measurements which have been carried out are described: neutron diffraction measurements on both cementite and ferrite phases in the bulk of the wires, and X-ray diffraction measurements on the ferrite phase at the surface. Neutron diffraction led to the average texture and the average phase microstresses in the axial direction in both the ferrite and the cementite phases. X-ray diffraction led to the total phase stress at the surface of the ferrite. By combining both results, it turned out to be possible to determine the macrostress at the surface of the wires and the total phase stress in the cementite as well. Additional measurements, performed after chemical thinning of the wires, finally led to some information about the stress gradients.

Keywords: High-carbon steel; Wire; Cementite; Residual stress; Microstress; Texture; Neutron diffraction; X-ray diffraction

\section{INTRODUCTION}

In this work, the residual stress and the texture in cold drawn wires of high carbon steel $(0.8 \mathrm{wt} \% \mathrm{C})$ has been studied. It is a two-phase material consisting of cementite $\left(\mathrm{Fe}_{3} \mathrm{C}\right)$ and ferrite lamellae. After wire

\footnotetext{
* Corresponding author.
} 
drawing, these lamellae are nearly parallel with the wire axis. The volume fraction of the cementite is $12 \%$.

The wires were drawn from a diameter of $3.25 \mathrm{~mm}$ to diameters of 1.22 and $0.59 \mathrm{~mm}$. The corresponding true strains are 1.96 and 2.59, respectively. After drawing, the spacing between the cementite lamellae is of the order of $100 \mathrm{~nm}$. The wire drawing operation introduces important residual stresses in these wires. They may have an important effect on the functional properties of the wire.

In the work reported in this paper, it has been attempted to measure the texture and the residual stresses in both the ferrite and the cementite phases. Both neutron and X-ray diffraction have been used. During data processing, the specific properties of each method have been exploited to generate as much information on the residual stresses as possible. A short theoretical treatise on internal stresses in two-phase materials is necessary before more details on the data processing can be given.

The goal of the present paper is to present this work to texture experts rather than to experts in residual stress analysis. Hence focus will be more on the general aspects of the problem and on the interpretation of the results, than on the fine details of the method, which have been described elsewhere (Van Acker et al., 1996; Van Acker, 1996). The work of Noyan and Cohen (1987) can for example be consulted for general reading on residual stress analysis.

\section{THEORY OF STRESSES IN TWO-PHASE MATERIALS}

\section{Introduction}

The various concepts needed for the analysis of stress distributions in two-phase materials are introduced using a step-by-step method, which first discusses a very simple case, then a more complex case, etc. In each stage, new concepts are introduced while maintaining the concepts introduced in the previous stages.

\section{Macrostress}

Before coming to these case studies, it is necessary to introduce the concept of the macrostress $\sigma^{\mathbf{M}}$. It is usually defined as the average stress in an 'intermediate volume' $V$. Such intermediate volume is small 
when compared to the wire as a whole, but large when compared to individual grains (in this case, cementite or ferrite lamellae). For the material at hand, an intermediate volume would have a typical size of about $2-5 \mu \mathrm{m}$. Hence

$$
\sigma^{\mathrm{M}}=\frac{\int_{V} \sigma \mathrm{d} V}{\int_{V} \mathrm{~d} V}
$$

The macrostress is position-dependent, since the intermediate volume can be located anywhere in the material.

\section{Case 1: No Residual Stresses}

The following thought experiment is carried out:

(i) Suppose that all stresses (local, macroscopic) in all phases are zero.

(ii) Apply a nominal stress $\sigma^{\mathrm{N}}=F / A$, in which $A$ is the cross section of the wire, and $F$ an applied force. The macrostress is then uniformly distributed over the cross section, and it is equal to the nominal stress (Fig. 1):

$$
\sigma^{\mathrm{M}}=\sigma^{\mathrm{N}}
$$

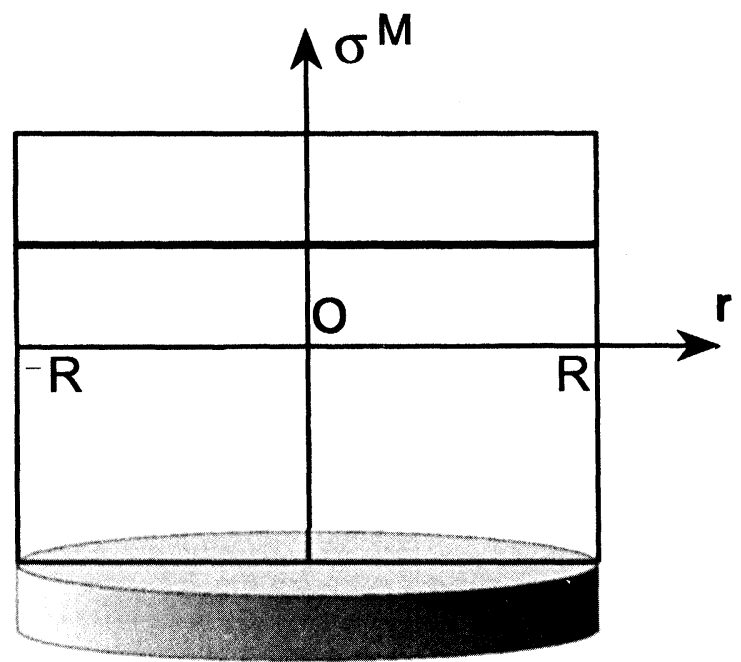

FIGURE 1 Stress distribution in a wire without residual stresses but with an applied load. 
(iii) At the microscopic level, the macrostress is felt as if it were an external stress. All local stresses are proportional to it:

$$
\sigma(\mathbf{x})=\mathbf{F}(\mathbf{x}) \sigma^{\mathrm{M}},
$$

in which the vector $\mathbf{x}$ is the position and the 4th order tensor $\mathbf{F}(\mathbf{x})$ is a stress transfer factor (called 'stress transfer coefficient' by Behnken and Hauk (1991)).

In principle, the position $\mathbf{x}$ also carries the information about phase and crystallite orientation (represented by the symbol $g$ ). This of course requires a detailed knowledge of the microstructure (including the crystallite orientations). In such case, it would theoretically be possible to calculate $\mathbf{F}(\mathbf{x})$ as well, using the finite element method in combination with anisotropic elasticity theory.

All this calls for some simplification. $\mathbf{F}(\mathbf{x})$ will be supposed to depend only on phase $\alpha$ and crystallite orientation $g$. As a result, the local stress will also depend only on $\alpha$ and $g$. Equation (3) can then be re-written as:

$$
\sigma^{\alpha}(g)=\mathbf{F}^{\alpha}(g) \sigma^{\mathrm{M}}
$$

The average of $\sigma^{\alpha}(g)$ over all phases and all $g$ must be equal to $\sigma^{\mathrm{M}}$.

The microstress is then defined as the difference of the local stress with the macrostress:

$$
\Delta^{\mathrm{e}} \sigma^{\alpha}(g)=\sigma^{\alpha}(g)-\sigma^{\mathrm{M}} .
$$

The average of this microstress over all phases and all $g$ must be equal to zero.

The symbol e indicates that this type of microstress is caused by the elastic interaction. Note that if the microstructure would be completely homogeneous (same phase, same crystallite orientation everywhere), the absence of any elastic interaction between different elements in the microstructure would make this kind of microstress zero. From Eqs. (4) and (5) it follows that

$$
\Delta^{\mathrm{e}} \sigma^{\alpha}(g)=\left(\mathbf{F}^{\alpha}(g)-\mathbf{I}\right) \sigma^{\mathbf{M}} .
$$


It is possible to average $\sigma^{\alpha}(g)$ and $\Delta^{\mathrm{e}} \sigma^{\alpha}(g)$ over all crystallite orientations of the phase $\alpha$. The resulting averages are called $\sigma^{\alpha}$ and $\Delta^{\mathrm{e}} \sigma^{\alpha}$, respectively:

$$
\sigma^{\alpha}=\int \sigma^{\alpha}(g) f^{\alpha}(g) \mathrm{d} g
$$

in which $f^{\alpha}(g)$ represents the ODF of phase $\alpha$, and

$$
\begin{aligned}
\Delta^{\mathrm{e}} \sigma^{\alpha} & =\int \Delta^{\mathrm{e}} \sigma^{\alpha}(g) f^{\alpha}(g) \mathrm{d} g \\
& =\sigma^{\mathrm{M}} \int\left(\mathbf{F}^{\alpha}(g)-\mathbf{I}\right) f^{\alpha}(g) \mathrm{d} g=\left(\mathbf{F}^{\alpha}-\mathbf{I}\right) \sigma^{\mathrm{M}},
\end{aligned}
$$

in which $\mathbf{F}^{\alpha}$ has been defined as follows:

$$
\mathbf{F}^{\alpha}=\int \mathbf{F}^{\alpha}(g) f^{\alpha}(g) \mathrm{d} g .
$$

$\sigma^{\alpha}$ is also called the phase stress of phase $\alpha$.

From the fact that the average of the microstresses over all phases and all crystal orientations (in the intermediate volume) is zero, it follows that (for a two-phase material):

$$
c^{\alpha} \Delta^{\mathrm{e}} \sigma^{\alpha}+c^{\beta} \Delta^{\mathrm{e}} \sigma^{\beta}=0,
$$

in which $c^{\alpha}$ and $c^{\beta}$ are the volume fractions of phase $\alpha$ and phase $\beta$. The average of all stresses over all phases and all crystal orientations (in the intermediate volume) must be equal to $\sigma^{\mathbf{M}}$, which leads to:

$$
c^{\alpha} \sigma^{\alpha}+c^{\beta} \sigma^{\beta}=\sigma^{\mathbf{M}} .
$$

Figure 2 illustrates the concepts which have been introduced so far (macrostress 'felt' in an intermediate volume, but caused by a nominal stress), phase stresses and microstresses.

\section{Case 2: Macroscopic Residual Stresses}

It is now assumed that residual stresses are present at macro-level. Figure 3 shows their distribution. As to the microscopic level, nothing is changed as compared to the previous case. This means that all 

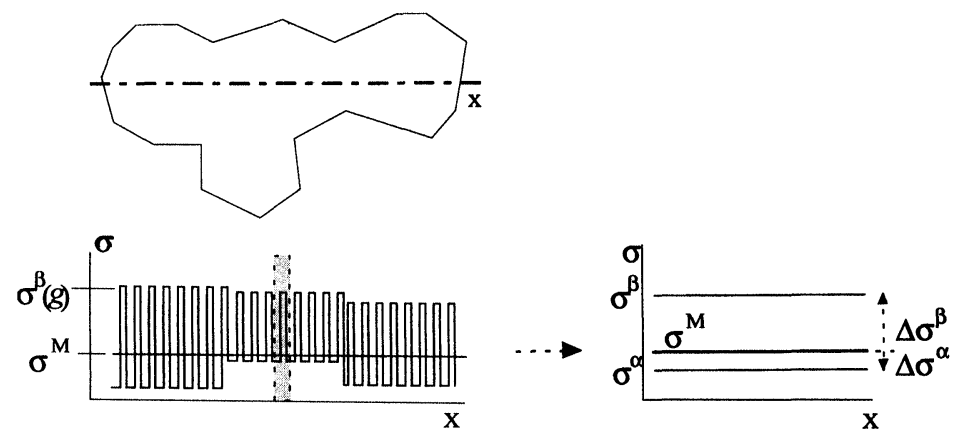

FIGURE 2 Schematic representation of macrostress $\sigma^{\mathrm{M}}$, total phase stresses $\sigma^{\alpha}(g)$ and $\sigma^{\beta}(g)$, and microstresses $\Delta \sigma^{\alpha}$ and $\Delta \sigma^{\beta}$. In the example of Case 1 , the microstresses are merely due to elastic interactions at the microscopical level.

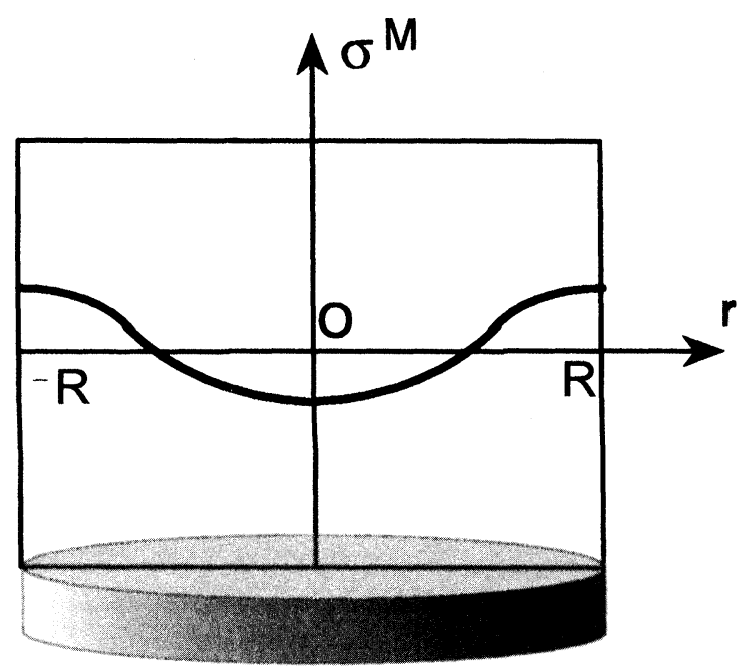

FIGURE 3 Distribution of macro-residual stresses in a wire.

concepts and equations introduced in the previous section for the microscopic level are still valid, the only change being that $\sigma^{\mathrm{M}}$ is not equal to an applied stress, but is a residual stress.

The macro-residual stress $\sigma^{\mathrm{M}}$ needs to be in equilibrium over the entire volume of the sample (Fig. 3). This leads to:

$$
\int \sigma^{\mathrm{M}} \mathrm{d} V=0
$$


$V$ is the volume of the entire wire. Suppose that $F^{\alpha}$ is constant throughout the wire. It then follows from Eq. (12) that

$$
\left(\mathbf{F}^{\alpha}(g)-\mathbf{I}\right) \int \sigma^{\mathrm{M}} \mathrm{d} V=\int\left(\mathbf{F}^{\alpha}(g)-\mathbf{I}\right) \sigma^{\mathrm{M}} \mathrm{d} V=0
$$

and from Eq. (6):

$$
\int \Delta^{\mathrm{e}} \sigma^{\alpha} \mathrm{d} V=0
$$

This is valid when $\mathbf{F}^{\alpha}$ is constant throughout the wire, which is true if texture and microstructure are homogeneously distributed.

\section{Case 3: Microscopic Residual Stresses}

In the previous sections, the only microstresses present were due to elastic interaction between the phases. As a result, all microstresses would become zero if the macrostress $\sigma^{\mathbf{M}}$ would be reduced to zero.

However, due to previous plastic deformation, it is possible that there are non-zero microstresses (different in each phase) even if $\sigma^{\mathbf{M}}=0$. They are called phase microstresses, and their symbol is $\Delta^{\mathrm{r}} \sigma^{\alpha}(g)$. When averaged over all crystallites of a particular phase, the symbol $\Delta^{\mathrm{r}} \sigma^{\alpha}$ is used.

The condition of equilibrium in an intermediate volume leads to:

$$
c^{\alpha} \Delta^{\mathrm{r}} \sigma^{\alpha}+c^{\beta} \Delta^{\mathrm{r}} \sigma^{\beta}=0,
$$

$c^{\alpha}, c^{\beta}$ being volume fractions of ferrite and cementite, respectively.

If the macrostress $\sigma^{\mathrm{M}}$ is not zero, the total microstress of a phase is equal to the sum of the phase microstress and the contribution of the elastic interaction:

$$
\Delta \sigma^{\alpha}(g)=\Delta^{\mathrm{r}} \sigma^{\alpha}(g)+\Delta^{\mathrm{e}} \sigma^{\alpha}(g)
$$

Note that

$$
\Delta \sigma^{\alpha}(g)=\sigma^{\alpha}(g)-\sigma^{\mathbf{M}},
$$

in which $\sigma^{\alpha}(g)$ is the total stress in a grain with orientation $g$ of the phase $\alpha$. 
Equations (16) and (17) can also be averaged over all crystallite orientations of a particular phase (in an intermediate volume). The averaged quantities are no longer functions of $g$ :

$$
\Delta \sigma^{\alpha}=\Delta^{\mathrm{r}} \sigma^{\alpha}+\Delta^{\mathrm{e}} \sigma^{\alpha}
$$

and

$$
\Delta \sigma^{\alpha}=\sigma^{\alpha}-\sigma^{\mathbf{M}}
$$

\section{Comment}

One could describe the internal stresses in a two-phase material by specifying the average phase stress in each phase, preferably as a function of location (position of the intermediate volume). The average of these stresses over the phases would then be the macrostress. The difference between the phase stress and the macrostress would then be the total microstress in a particular phase. However, for a correct interpretation of the result, it would be better if one could separate the total microstress in a phase into the elastic interaction part and the phase microstress as defined in the previous section. Only the phase microstress is a genuine microscopical residual stress, whereas the elastic interaction part is merely the reaction of the microstructure to the macrostress.

However, the parameters which can be measured depend to a large extent on the diffraction method which is used. By selecting the Bragg condition for one particular phase, the total stress $\sigma^{\alpha}$ in a particular phase is obtained when X-ray radiation is used. This will be the total phase stress at the surface of the sample if an ordinary X-ray generator is used. It can also be measured under the surface if synchrotron radiation would be used.

Spatial resolution is however much lower if neutron diffraction is used. In case of thin wires, the neutrons penetrate the whole sample. As a result, it is the volume average of $\sigma^{\alpha}$ which is obtained. From Eqs. (18) and (19), it follows that

$$
\sigma^{\alpha}=\Delta \sigma^{\alpha}+\sigma^{\mathrm{M}}=\Delta^{\mathrm{r}} \sigma^{\alpha}+\Delta^{\mathrm{e}} \sigma^{\alpha}+\sigma^{\mathrm{M}} .
$$

Then, since the volume average of $\sigma^{\mathrm{M}}$ is zero, and the volume average of $\Delta^{\mathrm{e}} \sigma^{\alpha}$ is (probably) zero (Eq. (14)), the volume average of $\sigma^{\alpha}$ will yield the volume average of the phase microstress $\Delta^{\mathrm{r}} \sigma^{\alpha}$. It can be 
concluded that, at least in the case of thin wires, neutron diffraction yields the volume average of the phase microstresses, and not the total stress in the phase.

\section{MEASUREMENT OF STRESSES IN WIRES}

The diffraction method for measuring residual stresses (Noyan and Cohen, 1987) is applied to thin wires, using monochromatic radiation. Let $\mathbf{Q}$ be the diffraction vector (the bisector between incident beam and diffracted beam, Fig. 4). The lattice spacing $d_{h k l}$ of a lattice plane $\{h k l\}$ which is normal to $\mathbf{Q}$ is measured. The strain in the direction $\mathbf{Q}$ is then given by:

$$
\varepsilon_{\mathbf{Q}}=\frac{d_{h k l}-d_{h k l}^{0}}{d_{h k l}^{0}}
$$

in which $d_{h k l}^{0}$ is the lattice spacing of a stress-free plane. Such measurement has to be done in several directions, characterised by the angles $\varphi$ and $\psi$ as defined in Fig. 4. The values of $\varepsilon_{\mathbf{Q}}$ for these various directions can then be used to derive the residual stress. This

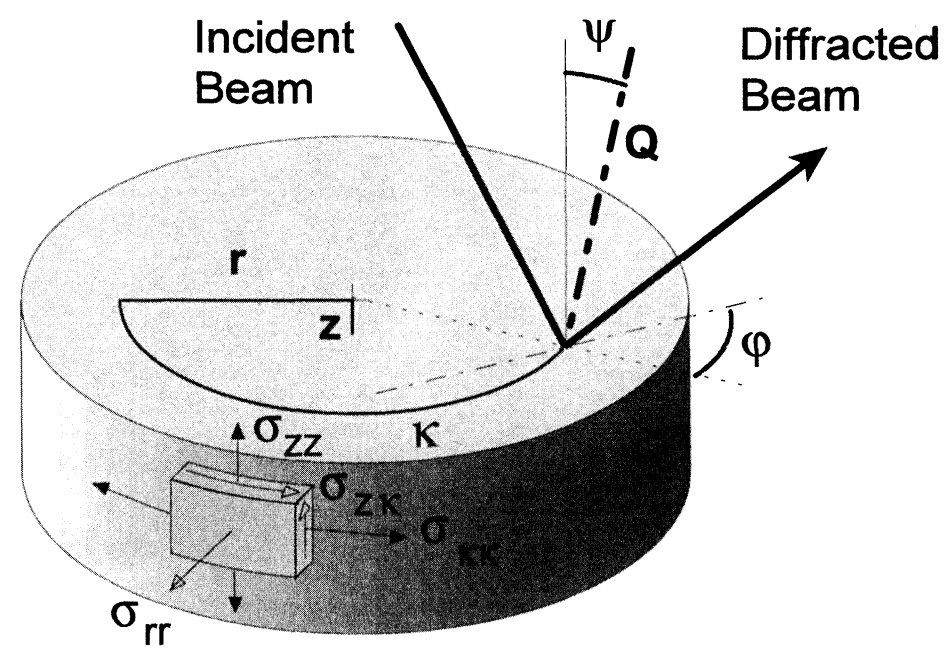

FIGURE 4 Definition of the stress components in a cylindrical coordinate system and definition of the angles $\psi$ and $\varphi$ in the neutron diffraction experiment. 
of course requires equations, of which the most important ones will now be given.

Let $\varepsilon_{\varphi \psi}(r, \varphi)$ be the strain in the direction $\mathbf{Q}$ (characterised by $\psi$ ), at the position $(r, \kappa)$. The stresses and strains in the wires have a cylindrical symmetry. It means that, if cylindrical co-ordinates are used (see Fig. 4 for their definition), stresses and strains would be independent of $\kappa$ and $z$. In such case, the relationship between the strain as measured by the diffraction experiment and the angles $\varphi$ and $\psi$ (also defined in Fig. 4) can be expressed as (Van Acker et al., 1996; Van Acker, 1996):

$$
\begin{aligned}
\varepsilon_{\varphi \psi}(r)= & \frac{1}{2} s_{2}\left(\sigma_{r r}^{\alpha} \cos ^{2} \varphi+\sigma_{r \kappa}^{\alpha} \sin 2 \varphi+\sigma_{\kappa \kappa}^{\alpha} \sin ^{2} \varphi-\sigma_{z z}^{\alpha}\right) \sin ^{2} \psi+\frac{1}{2} s_{2} \sigma_{z z}^{\alpha} \\
& +s_{1}\left(\sigma_{r r}^{\alpha}+\sigma_{\kappa \kappa}^{\alpha}+\sigma_{z z}^{\alpha}\right)+\frac{1}{2} s_{2}\left(\sigma_{r z}^{\alpha} \cos \varphi+\sigma_{\kappa z}^{\alpha} \sin \varphi\right) \sin 2 \psi
\end{aligned}
$$

$\varepsilon_{\varphi \psi}(r)$ can be measured for $r=R$ (at the surface) when X-ray diffraction is used. Only $\bar{\varepsilon}_{\psi}$, the volume average of $\varepsilon_{\varphi \psi}(r)$, can be measured when neutron diffraction is used.

It can be shown that (Van Acker et al., 1996; Van Acker, 1996):

$$
\bar{\varepsilon}_{\psi}=\left(\frac{1}{2} s_{2} \cos ^{2} \psi+s_{1}\right) \overline{\sigma_{z z}^{\alpha}}
$$

using the stress equilibrium equations and the assumption that $\sigma_{r r}^{\alpha}=0$ at the surface of the wire. This assumption has been checked on the basis of measured data (Van Acker et al., 1996; Van Acker, 1996).

It is reminded that the average phase stress $\overline{\sigma_{z z}^{\alpha}}$ is equal to $\overline{\Delta^{\mathrm{r}} \sigma_{z z}^{\alpha}}$, the volume average of the phase microstress $\Delta^{\mathrm{r}} \sigma^{\alpha}$. Equation (23) leads to:

$$
\bar{d}_{h k l, \psi}=\left(\frac{1}{2} s_{2}+s_{1}-\frac{1}{2} s_{2} \sin ^{2} \psi\right) d_{h k l}^{0} \overline{\Delta^{\mathrm{r}} \sigma_{z z}^{\alpha}}+d_{h k l}^{0},
$$

in which $\bar{d}_{h k l, \psi}$ is the average lattice spacing of the $\{h k l\}$ plane, as seen by the neutrons and $\frac{1}{2} s_{2}$ and $s_{1}$ are the diffraction elastic constants (formerly called ' $\mathrm{X}$-ray elastic constants').

The average phase microstress have been estimated by fitting the above equation to measured $\bar{d}_{h k l, \psi}-\psi$ data.

However, the diffraction elastic constants which appear in Eq. (24) depend in principle on the texture. As a result, they may be functions of $\psi$, in which case nonlinear $d-\sin ^{2} \psi$ curves are obtained. This case 
can be dealt with by using the Reuss, Voigt or Kröner assumptions in combination with the ODF of the phase (Van Houtte and De Buyser, 1993). This special method has been used for the treatment of the residual stresses in the ferrite phase of the wires. This method could not be used for the cementite phase since the ODF of this phase could not be obtained. The diffraction elastic constants reported by Hanabusa et al. (1969) have been used instead.

\section{EXPERIMENTAL SET-UP (NEUTRON DIFFRACTION)}

The $\mathrm{C} 2$ high resolution powder diffractometer at AECL, Chalk River, Canada was used. Figure 5 shows a schematic representation of the set-up. The neutron wavelength was $2.3654 \AA$. Higher order contamination was suppressed by a pyrolytic graphite filter.

Intensities were recorded for a $2 \theta$-range of $41-121^{\circ}$ using an 800 channel PSD. A sample of about $1 \mathrm{~cm}^{3}$ was prepared by stacking pieces of wires together (Fig. 6). The wire axes were in the scattering plane. The angle between the wire axis and the incident beam was varied from $0^{\circ}$ to $180^{\circ}$ in steps of $20^{\circ}$. A diffraction pattern was obtained for each of these sample positions. Counting times up to $4 \mathrm{~h}$ were required to detect the weak cementite peaks.

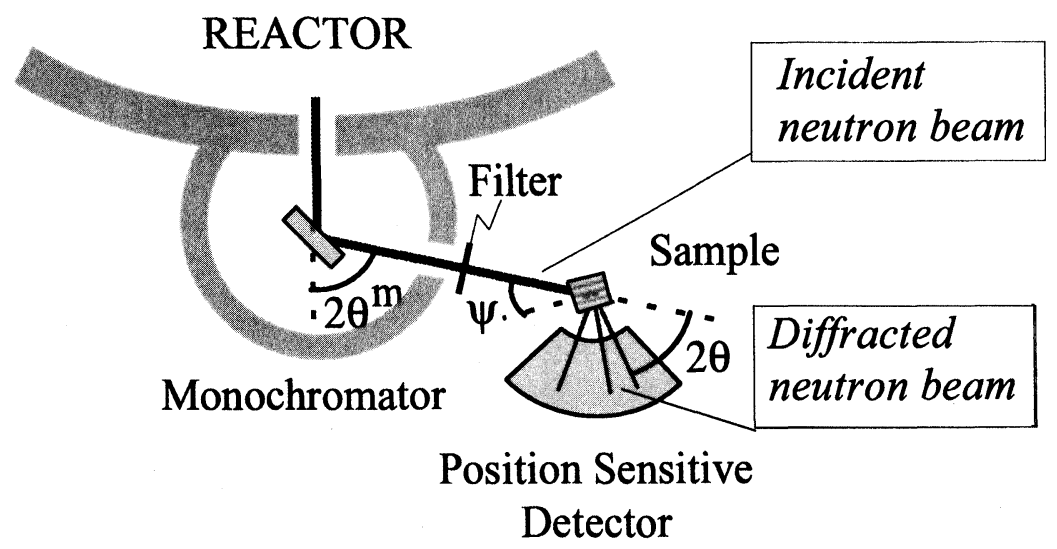

FIGURE 5 Schematic representation of the set-up of the neutron diffraction experiment. 


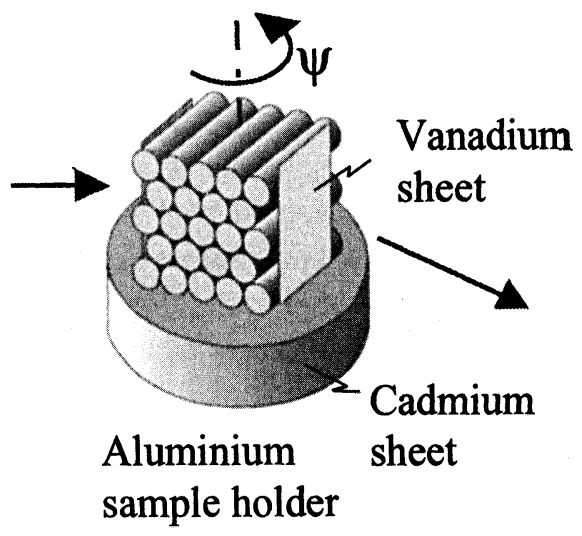

FIGURE 6 Stack of wires used in neutron diffraction experiment.

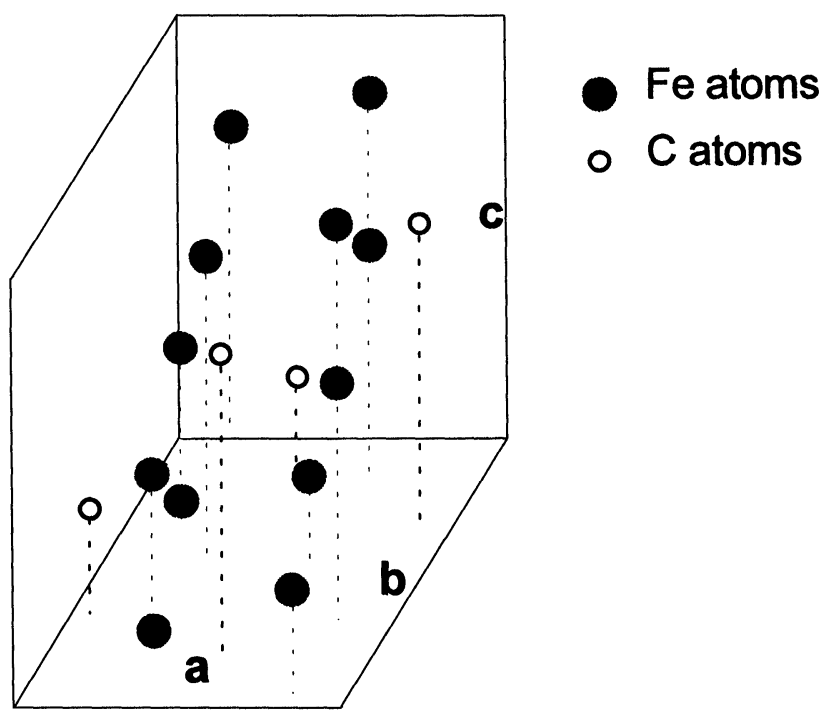

FIGURE 7 Atom positions in the unit cell of cementite.

\section{RESULTS}

\section{Cementite}

Cementite has an orthorhombic unit cell (Villars and Calvert, 1991). It contains $12 \mathrm{Fe}$-atoms and $4 \mathrm{C}$-atoms (Fig. 7). The present authors have 
again determined the lattice parameters by means of neutron diffraction on a stress-free wire. The results are given in Table I.

Figure 8 shows examples of the neutron diffraction data which have been obtained for ferrite and cementite. The reference sample is a stress-free sample. The sample marked 'Wire $\varepsilon=1.96$ ' has been cold drawn. It is clear that the latter shows considerable line broadening. The reason for it is unclear, but it has been suggested by Van Acker et al. (1996) that the most likely cause is the size of the diffraction domain, which is quite small in cold drawn wires. This is due to the small interlamellar spacing, which is of the order of $100 \mathrm{~nm}$.

TABLE I Lattice parameters of cementite obtained by neutron diffraction

\begin{tabular}{lcc}
\hline & Lattice par. $(\AA)$ & Uncertainty (St. Dev.) $(\AA)$ \\
\hline$a$ & 5.0816 & 0.0007 \\
$b$ & 6.7446 & 0.0016 \\
$c$ & 4.5206 & 0.0007 \\
\hline
\end{tabular}

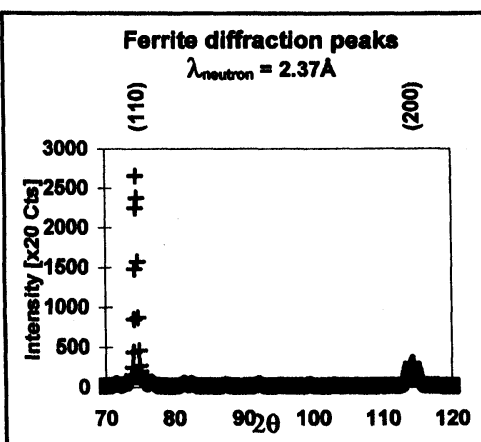

Cementite diffraction peaks

$\lambda_{\text {nowaro }}=2.37 \AA$

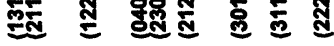
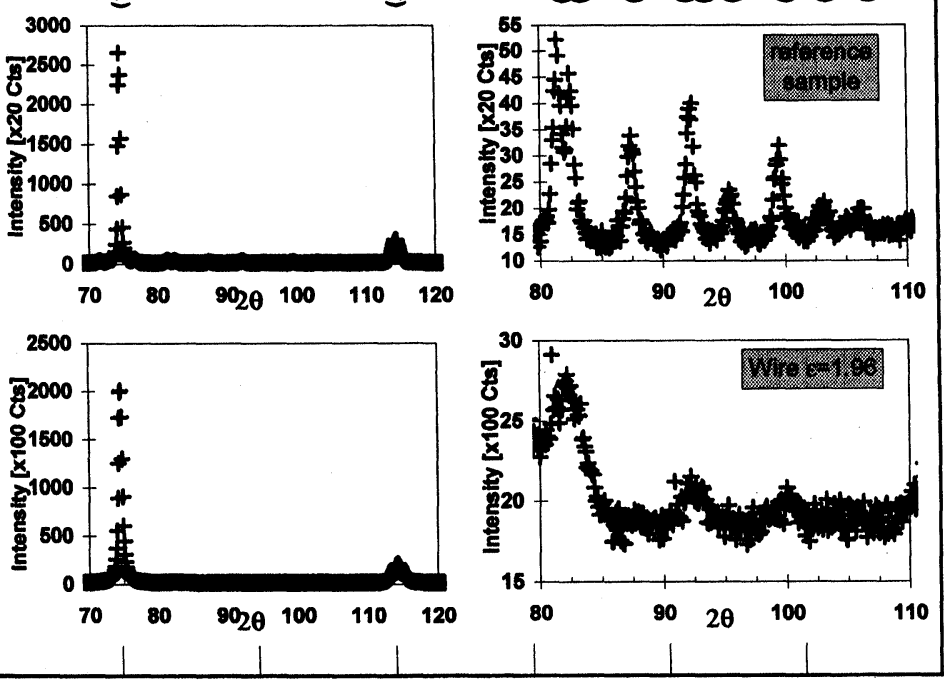

FIGURE 8 Details of the diffraction patterns of the stress-free sample (reference sample) and of the sample which was cold drawn until $\varepsilon=1.96$. 


\section{Texture}

Due to the large penetration depth, neutron diffraction can only measure the average texture of the wires. This must then necessarily be a fibre texture because of the cyclic symmetry of the wire. Instead of showing a complete pole figure, it is then sufficient to show the intensity obtained at an angle $\chi$ from the direction of the wire axis. Figure 9 shows such result for the cold drawn wire with $\varepsilon=1.96$.

The intensities are corrected for background and then normalised. The data indicate that the texture in the ferrite phase can completely be described by a $\langle 110\rangle$-fibre texture component. The texture of the cementite is more complicated.

The ODF of the ferrite phase has been obtained by first generating a complete pole figure from the data represented in Fig. 6 (assuming a perfect rotational symmetry around the wire axis), and then applying standard ODF software on the resulting pole figures.

Figure 10(a) shows the $\{110\}$-pole figure generated from the neutron diffraction data for the ferrite phase. For comparison, a $\{110\}-$ pole figure has also been measured at the surface of the wire (Fig. 10(b)). This measurement has been processed assuming cyclic
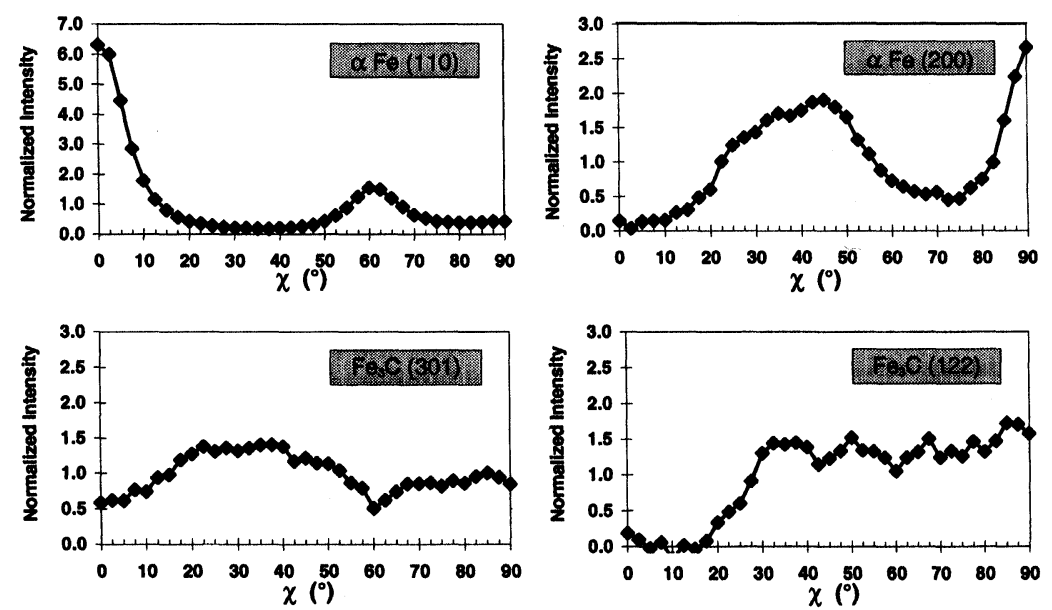

FIGURE 9 Neutron diffraction intensity versus $\chi$ for some reflections. $\chi$ is the angle between the wire axis to the diffraction vector. The texture measurements were done with neutron diffraction, $\lambda=2.37 \AA$, at $2 \theta=71.5^{\circ}$ for $\alpha \mathrm{Fe}(110), 111.5^{\circ}$ for $\alpha \mathrm{Fe}$ (200), $96.5^{\circ}$ for $\mathrm{Fe}_{3} \mathrm{C}(301)$ and $84.4^{\circ}$ for $\mathrm{Fe}_{3} \mathrm{C}$ (122). 

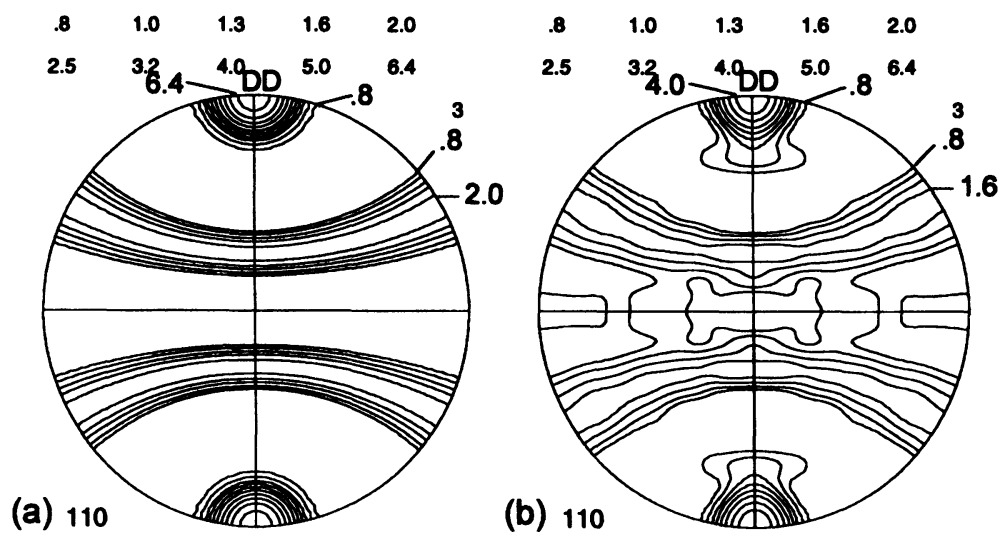

FIGURE 10 (a) $\{110\}$-pole figure recalculated from the ODF of the ferrite phase, as derived from neutron diffraction data (Fig. 9). (b) $\{110\}$-pole figure recalculated from the ODF of the surface texture of the wire (ferrite phase), as obtained from X-ray diffraction measurements.

texture symmetry (Van Acker et al., 1994; Van Acker, 1996), not fibre texture symmetry, since the latter can only be expected to exist in the centre of the wire. In spite of a certain deviation from a perfect rotational symmetry, this pole figure compares well to the neutron diffraction pole figure represented in Fig. 10(a).

\section{Stress Measurements}

Figure 11 shows typical $d-\sin ^{2} \psi$ results obtained by neutron diffraction. Such data are then analysed by the methods described above in order to yield the phase microstresses in the two phases. Table II gives an overview of the results obtained. It is seen that the phase microstresses in the cementite phase are quite high and tensile. In the ferrite, they are moderate and compressive.

Figure 12 shows the results obtained by means of X-ray diffraction at the surface of the wires (using monochromatic $\mathrm{K} \alpha \mathrm{Cr}$-radiation). This method could only be used for the ferrite phase, since the signal coming from the cementite was too weak. Figure 12 also shows the definition of the angles $\varphi^{*}$ and $\psi^{*}$, which is different from the angles $\varphi$ and $\psi$ used in the neutron diffraction experiments. Table III shows the results. 

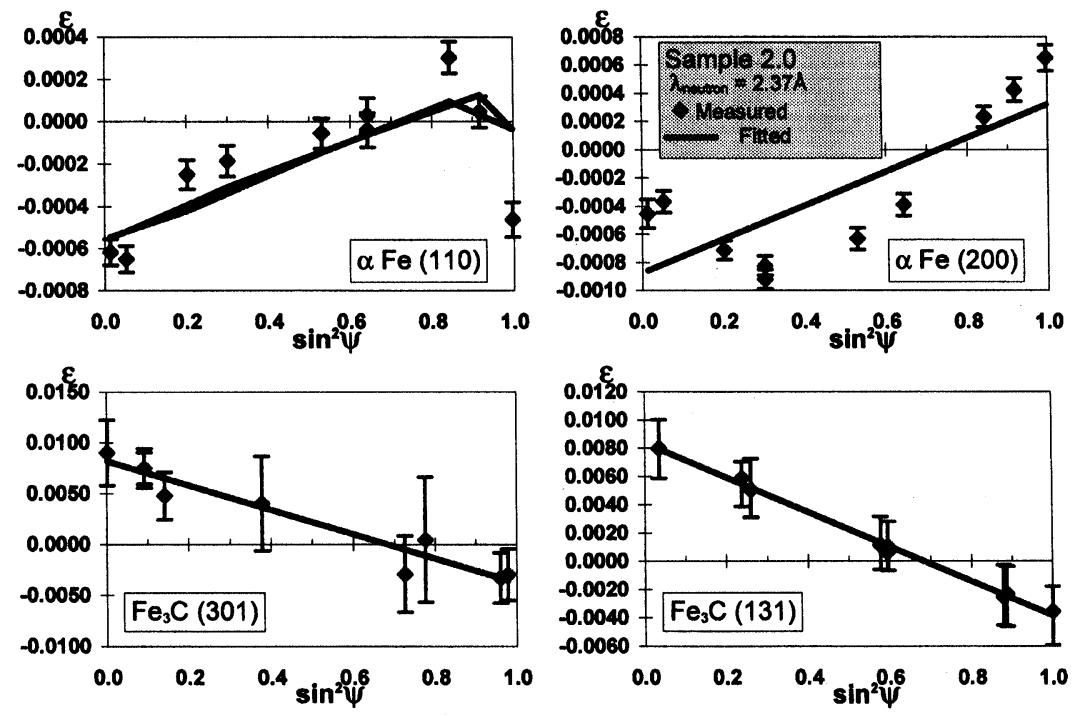

FIGURE $11 \varepsilon-\sin ^{2} \psi$ curves for cementite and ferrite diffraction planes as measured with the neutron diffraction technique. The error bars give the $95 \%$ confidence limit on the fit between the model used for the $\varepsilon-\sin ^{2} \psi$ relationship and the measured data.

TABLE II Average axial phase microstresses obtained by means of neutron diffraction for cold drawn wires. The $95 \%$ confidence limit on the fit between the model $\left(d-\sin ^{2} \psi\right.$ curve) and the measured data is given between brackets

\begin{tabular}{lccccc}
\hline Sample plane & \multicolumn{2}{c}{$\varepsilon=1.96$} & & \multicolumn{2}{c}{$\varepsilon=2.59$} \\
\cline { 2 - 3 } \cline { 5 - 6 } & $\mathrm{MPa}$ & $(\mathrm{MPa})$ & & $\mathrm{MPa}$ & $(\mathrm{MPa})$ \\
\hline$\alpha \mathrm{Fe}(110)$ & -152 & $(30)$ & & -122 & $(33)$ \\
$\alpha \mathrm{Fe} \mathrm{(200)}$ & -127 & $(36)$ & & -114 & $(32)$ \\
$\mathrm{Fe}_{3} \mathrm{C}(301)$ & 2270 & $(310)$ & & 1730 & $(170)$ \\
$\mathrm{Fe}_{3} \mathrm{C}(131)$ & 2050 & $(110)$ & & 1770 & $(50)$ \\
\hline
\end{tabular}

Some other measurements have been done on the $\varepsilon=1.96$ sample after removal of surface layer by etching, reducing the diameter to $90 \%$ and $70 \%$, respectively. The values of the phase microstresses were not significantly different, but there were important changes of the total stress in the ferrite phase (see below). 


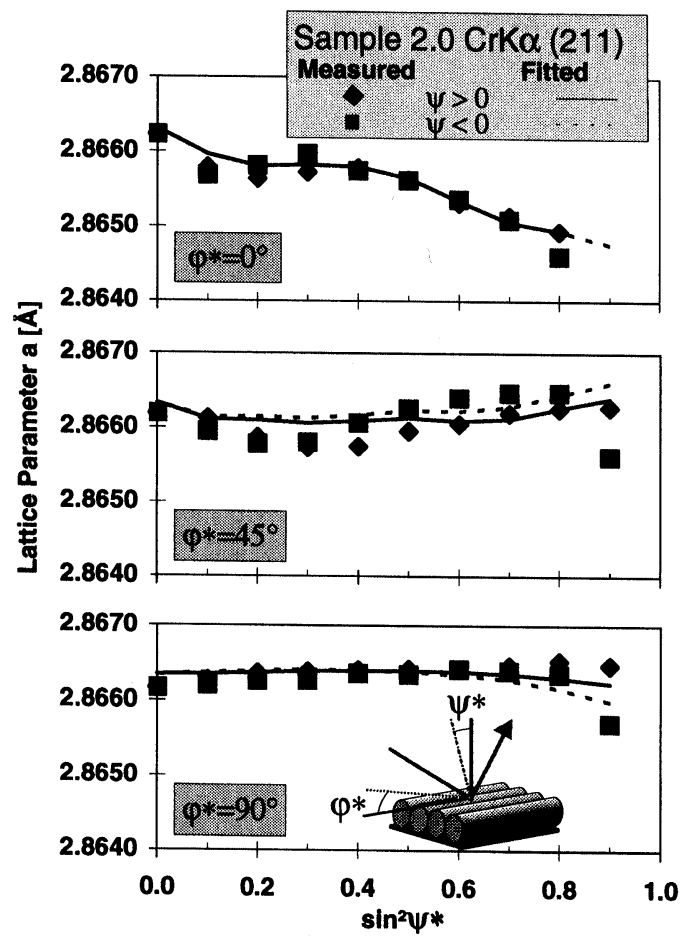

FIGURE 12 X-ray stress measurements on the $\alpha \mathrm{Fe}$ (211) diffraction peak, as represented by $d-\sin ^{2} \psi^{*}$ curves. The definition of $\varphi^{*}$ and $\psi^{*}$ is different as in the case of the neutron diffraction set-up.

TABLE III Total stress of the ferrite phase at the surface, measured by X-ray diffraction

\begin{tabular}{ccccccc}
\hline Sample & \multicolumn{2}{c}{$\sigma_{z z}^{\alpha}(\mathrm{MPa})$} & \multicolumn{2}{c}{$\sigma_{z \kappa}^{\alpha}(\mathrm{MPa})$} & \multicolumn{2}{c}{$\sigma_{\kappa \kappa}^{\alpha}(\mathrm{MPa})$} \\
\hline$\varepsilon=1.96$ & -114 & $(8)$ & -94 & $(16)$ & -56 & $(23)$ \\
$\varepsilon=2.59$ & -156 & $(16)$ & -38 & $(38)$ & -48 & $(53)$ \\
\hline
\end{tabular}

Note that the total phase stress is given by (Eqs. (4), (9) and (20)):

$$
\sigma^{\alpha}=\sigma^{\mathrm{M}}+\Delta^{\mathrm{r}} \sigma^{\alpha}+\Delta^{\mathrm{e}} \sigma^{\alpha}=\mathbf{F}^{\alpha} \sigma^{\mathrm{M}}+\Delta^{\mathrm{r}} \sigma^{\alpha} .
$$

The axial component of the phase microstress $\Delta^{\mathrm{r}} \sigma^{\alpha}$ is known from the neutron diffraction measurement. The axial component of the total phase stress $\sigma^{\alpha}$ is known at the surface from the X-ray diffraction 


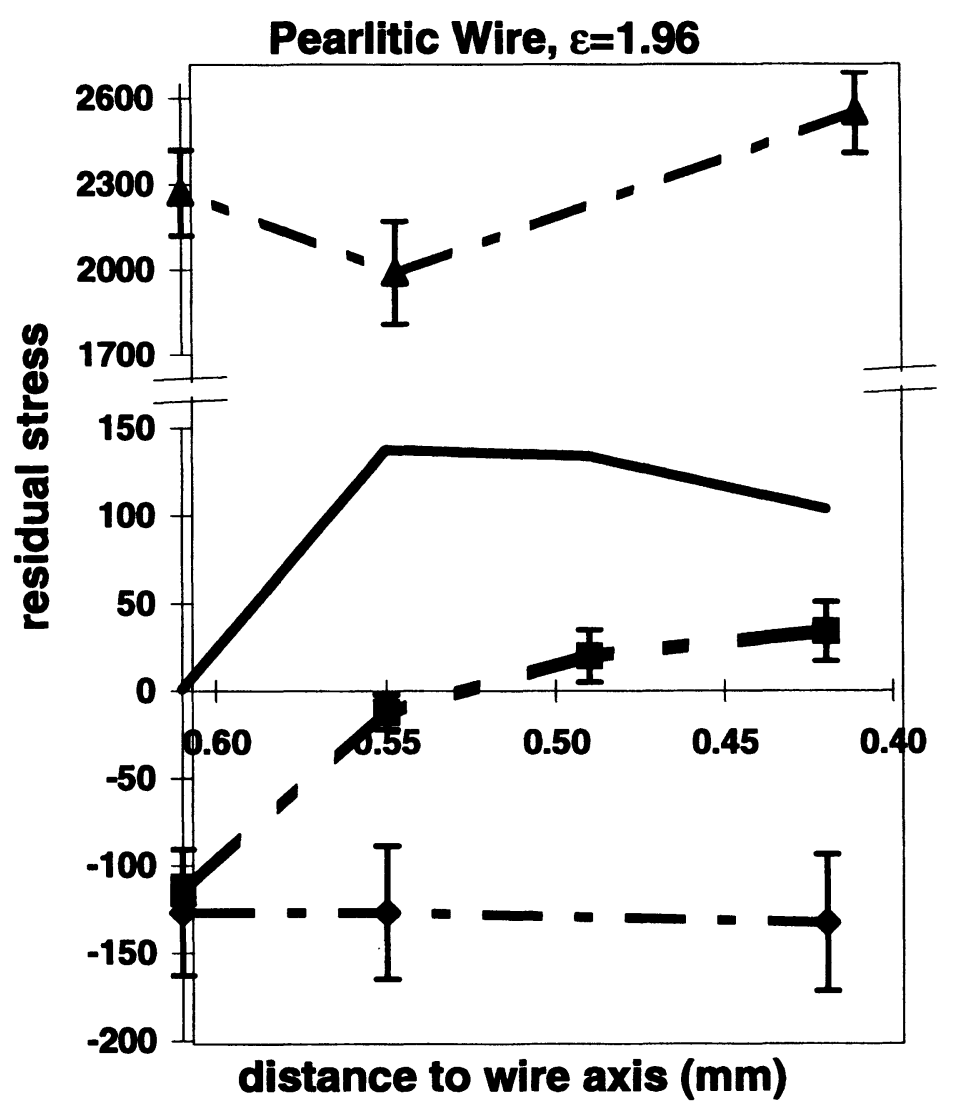

$\Delta$ phase microstress of $\mathrm{Fe}_{3} \mathrm{C}$ (neutron diffraction measurement)

- macrostress (calculated with correction for relaxation)

- phase stress of $\alpha$-Fe (X-ray diffraction measurement)

- phase microstress of $\alpha-F e$ (neutron diffraction measurement)

FIGURE 13 Stress results for the wire drawn to $\varepsilon=1.96$ obtained by combining the neutron and X-ray diffraction techniques. The surface of the wire is at the point ' $0.61 \mathrm{~mm}$ ' on the scale of the horizontal axis. 
measurements. It then follows from the above equation that the macrostress $\sigma^{\mathrm{M}}$ at the surface could be calculated if the elastic interaction factor $\mathbf{F}^{\alpha}$ would be known. So an in situ tensile test on a sample in an X-ray stress goniometer was set up in order to measure it. First the macrostress was measured without applying an external load. Then an external load was applied, thus generating a known increase in macrostress $\Delta \sigma^{\mathrm{M}}$. The change in phase stress was then measured. Equation (25) shows that this increase must be equal to $\mathbf{F}^{\alpha} \Delta \sigma^{\mathbf{M}}$. Since $\Delta \sigma^{\mathrm{M}}$ is known, $\mathbf{F}^{\alpha}$ can now be estimated. It was found that $\mathbf{F}^{\alpha}=\mathbf{0 . 9 4 4}$ for the axial stress in the ferrite phase. $\sigma^{\mathrm{M}}$ can now be solved from Eq. (25).

This procedure was repeated after etching down parts of the wire which was cold drawn until $\varepsilon=1.96$. Each time the axial phase stress was measured at the surface. A correction factor for the partial relaxation was used. Figure 13 shows the result. It is believed to be a coincidence that the axial component of the macrostress is nearly zero at the surface. It reaches a value of $150 \mathrm{MPa}$ at a depth of $0.06 \mathrm{~mm}$ under the surface, after which it starts to decrease. The evolution of the total axial phase stress in the cementite is also shown. It has been derived from the equilibrium (Eq. (11)).

\section{CONCLUSIONS}

- Neutron diffraction makes it possible to measure the phase microstress in both the ferrite and the cementite phases.

- Tensile stresses of about $2000 \mathrm{MPa}$ have been found in the cementite.

- The through-thickness gradient of the phase microstresses is not significant.

- Combination with X-ray measurements at the surface makes it possible to find the macrostress, the phase microstress and the elastic interaction term.

\section{Acknowledgements}

This work has been made possible through the financial support through the Belgian programme on Interuniversity Poles of Attraction 
initiated by the Belgian State, Prime Minister's Office, Science Policy Programming (Contract IUAP IV P4/33). Two of the authors (K.V.A. and P.V.H.) gratefully acknowledge the financial support of the N.F.W.O./F.W.O. The authors also thank the company N.V. Bekaert for providing test material. The specimens were assembled for neutron diffraction in mounts made by L. McEwan at AECL. R. Donaberger and $\mathrm{D}$. Tennant assisted with the neutron powder diffractometer.

\section{References}

Behnken, H. and Hauk, V. (1991). Die Bestimmung der Mikroeigenspannungen und ihre Berücksichtigung bei der röntgenographische Ermittlung der MakroEigenspannungen in mehrphasigen Materielen. In Werkstoffkunde, Beitrage zu de Grundlagen und zur interdisziplinären Anwendung, edited by P. Mayr, O.Vöhringer and $\mathrm{H}$. Wohlfahrt, pp. 141-150. Oberursel (Germany): DGM.

Hanabusa, T., Fukura, J. and Fujiwara, H. (1969). X-ray stress measurements on the cementite phase in steels. Bull. JSME 12, 931-939.

Noyan, I.C. and Cohen, J.B. (1987). Residual Stress. New York: Springer.

Van Acker, K., Van Houtte, P. and Aernoudt, E. (1994). Wire geometry correction of texture and stress measurements on wires. In Proc. 10th Intntl. Conf. on Textures of Materials (ICOTOM 10), Clausthal, Germany (20-24/9/1993), edited by H.J. Bunge, Materials Science Forum 157-162, 2075-2082.

Van Acker, K. (1996). Internal stress states in cold worked metals and in metal matrix composites. (Ph.D. thesis). Leuven (Belgium): Department MTM, K.U. Leuven.

Van Acker, K., Root, J., Van Houtte, P. and Aernoudt, B. (1996). Neutron diffraction measurements of the residual stress in the cementite and the ferrite phases of colddrawn steel wires. Acta Mater. 44, 4253-4259.

Van Houtte, P. and De Buyser, L. (1993). Overview no. 103: The influence of crystallographic texture on diffraction measurements of residual stresses. Acta metall. mater. 41, 323-336.

Villars, P. and Calvert, C. (1991). Pearson's Handbook of Crvstallographic Data from Intermetallic Phases, p. 1894. Metals Park (Ohio): ASM. 